\title{
Space Weathering Investigations Enabled by NASA's Virtual Heliophysical Observatories (Invited Talk)
}

John F. Cooper, Joseph H. King, Natalia E. Papitashvili, Nand Lal, Edward C. Sittler, Steven J. Sturner, Howard K. Hills, Alexander S. Lipatov, Tamara J. Kovalick, Rita C. Johnson, Robert E. McGuire, Thomas W. Narock, and Adam Szabo NASA Goddard Space Flight Center, Greenbelt, Maryland, U.S.A.

Thomas P. Armstrong, Jerry W. Manweiler, and J. Douglas Patterson Fundamental Technologies, LLC, Lawrence, Kansas, U.S.A.

Robert B. McKibben, University of New Hampshire, Durham, New Hampshire, U.S.A.

Structural and chemical impact of the heliospheric space environment on exposed planetary surfaces and interplanetary dust grains may be generally defined as space weathering [Cooper et al., 2010]. In the inner solar system, from the asteroid belt inwards towards the Sun, the surface regolith structures of airless bodies are primarily determined by cumulative meteoritic impacts over billions of years, but the molecular composition to meters in depth can be substantially modified by irradiation effects. Plasma ions at $\mathrm{eV}$ to $\mathrm{keV}$ energies may both erode uppermost surfaces by sputtering, and implant or locally produce exogenic material, e.g. $\mathrm{He}-3$ and $\mathrm{H} 2 \mathrm{O}$, while more energetic ions drive molecular change through electronic ionization. Galactic cosmic ray ions and more energetic solar ions can impact chemistry to meters in depth. High energy cosmic ray interactions produce showers of secondary particles and energetic photons that present hazards for robotic and human exploration missions but also enable detection of potentially useable resources such as water ice, oxygen, and many other elements. Surface sputtering also makes ejected elemental and molecular species accessible for in-situ compositional analysis by spacecraft with ion and neutral mass spectrometers. Modeling of relative impacts for these various space weathering processes requires knowledge of the incident species-resolved ion flux spectra at plasma to cosmic ray energies and as integrated over varying time scales.

Although the main drivers for investigations of these processes come from NASA's planetary science and human exploration programs, the NASA heliophysics program provides the requisite data measurement and modeling resources to enable specification of the field \& plasma and energetic particle irradiation environments for application to space weather and surface weathering investigations. The Virtual Heliospheric Observatory (VHO), Virtual Energetic Particle Observatory (VEPO), Lunar Solar Origins Exploration (LunaSOX), and Space Physics Data Facility (SPDF) services now provide a wide range of inner heliospheric spacecraft data that can be applied to space weathering of potential exploration destinations including the Moon, asteroids, and the moons of Mars, as well to radiation hazard assessment for the spacecraft and human explorers. For example, the new VEPO service for time-averaging of multi-source ion flux spectra enables the specification of composite flux spectra from a variety of ongoing and 
legacy missions for applications to surface interaction modeling. Apollo to Artemis data resources of LunaSOX enable specific space weathering investigations for the Moon, while VHO more generally covers the space field and plasma environments of the inner and outer solar system from the sunward-most perihelia of the twin Helios spacecraft to the ongoing heliosheath passages of the twin Voyagers. Composite multi-source spectra from VEPO can also be applied to the continuing compilation of accumulated 1-AU fluence spectra, mostly contributed by solar wind plasma and energetic particle events, for determination of time-averaged particle compositional and kinetic energy output from the Sun and for modeling of long-term irradiation impacts on planetary surfaces.

Reference: Cooper, J. F., R. E. Hartle, E. C. Sittler Jr., R. M. Killen, S. J. Sturner, C. Paranicas, M. E. Hill, A. M. Rymer, P. D. Cooper, D. Pascu, R. E. Johnson, T. A. Cassidy, T. M. Orlando, K. D. Retherford, N. A. Schwadron, R. I. Kaiser, F. Leblanc, L. J. Lanzerotti, C. J. Alexander, H. B. Garrett, A. R. Hendrix, and W. H. Ip, Space Weathering Impact on Solar System Surfaces and Mission Science, Community White Paper submitted to Solar and Space Physics Decadal Survey (Heliophysics), Space Science Board, National Academies of Science, Nov. 12, 2010.

Abstract submitted for $11^{\text {th }}$ Annual International Astrophysics Conference, Palm Springs, California, March 18-23, 2012. 